\begin{tabular}{cc}
\hline International Journal of Advanced Astronomy, $7(2)(2019) 39-48$ \\
SPC \\
International Journal of Advanced Astronomy \\
Website: www.sciencepubco.com/index.php/IJAA \\
Research paper
\end{tabular}

\title{
Applying He's variational iteration method to FRW cosmology
}

\author{
V. K. Shchigolev * \\ Department of Theoretical Physics, Ulyanovsk State University, 42 L. Tolstoy Str.,Ulyanovsk 432000, Russia \\ *Corresponding author E-mail: vkshch@yahoo.com
}

\begin{abstract}
This work is devoted to the investigation of Friedmann-Robertson-Walker (FRW) cosmological models with the help of the so-called Variational Iteration Method (VIM). For this end, we briefly recall the main equations of the cosmological models and the basic idea of VIM. In order to approbate the VIM in FRW cosmology and demonstrate the main steps in solving by this method, we consider the test example of the universe with dust for which the exact solution of the model is known. Then, a solution for the spatially flat FRW model of the universe filled with the dust and quintessence is obtained when the exact analytic solution cannot be found. A comparison of our solution with the corresponding numerical solution shows that it is of a high degree of accuracy. Moreover, the Dynamical System Analysis to the dynamics of the homogeneous and isotropic FRW universes is used as a special case of generalized Lotka-Volterra system where the competitive species are the barotropic fluids filling the Universe. With the help of VIM, we have found the iterative formulae for the density parameters of the cosmological analog of the generalized Lotka-Volterra set of equations. All solutions illustrated graphically by means of Maple software.
\end{abstract}

Keywords: Approximate Solution; FRW Cosmological Models; Dynamical System Analysis; Variational Iteration Method.

\section{Introduction}

This document can Cosmology is one of the most actual areas of research in the field of theoretical physics. Modern and generalized cosmological theories today are also actively supported by the experimental results of the observational constraints of the Universe. So, the relatively recent discovery of the present acceleration of our Universe is strongly supported by type Ia supernovae, cosmic microwave background radiation, and Sloan Digital Sky Survey [1]-[6]. This acceleration is caused by the fact that most of the matter consists of dark energy (DE), which is an exotic energy with negative pressure. Unfortunately, the nature of the DE is still unknown. In this regard, it is worth mentioning that one of the main problems in theories of gravity is the difficulty of finding analytical solutions due to the highly nonlinear nature of the equations of the gravitational field, and therefore comparison with observations cannot be easily implemented. Therefore, it is important that other methods be effectively used to solve such equations, at least in approximate form.

This problem becomes especially complex in the presence of various non-linear sources of gravity. Under these conditions, various approximate solution methods can be used, such as the weak field approximation in the General Theory of Relativity [7], the slow-roll approximation in inflationary cosmology $[8,9]$, etc. It should be noted that in such approximations, one has to ignore some of the terms in the main equations of the theory, that yields losing the universality of the solutions obtained. The basic equation governing the dynamics of cosmic evolution is known as the Friedmann equation. Since this equation applies to many cosmological models, any approach to its solution is always of great interest (see, for example, $[10,11]$ and references therein).

Early, we have studied some cosmological models in Friedmann-Robertson-Walker space-time by means of the so-called Homotopy Perturbation Method [12,13]. First, we considered the specific example in order to approbate the HPM in cosmology and present the main steps in solving the Friedmann equation by this method [14]. Then, we obtained a solution for the spatially flat FRW model of the universe filled with the dust and quintessence. A comparison of our solutions with the corresponding numerical solutions shows that it is of a high degree of accuracy. Moreover, we have applied HPM to some other problems of General Relativity and cosmology [15]-[17]. Variational iteration method (VIM) is another well-known analytical approach for solving the nonlinear equations [18]-[20]. Its greatest advantages are that the method is meshless, the solution is an explicit function, and the iterations converge to the exact solution very rapidly [21]-[23]. We have used this method in some problems of General Relativity and cosmology (see, for example, [24, 25]). The purpose of the present paper is the application of He's VIM in obtaining the approximations for the Friedmann equation in FRW cosmology.

The well known alternative method to investigate the set of cosmological equations is the Dynamical System Analysis (DSA). The approach of this method is to find the numerical solutions and help in understanding the qualitative behavior of a given physical system [26]-[28]. For this purpose, the effective form of cosmological field equations describing the whole cosmic evolution history in a homogeneous and isotropic cosmological background is constructed as the autonomous system of the first order dynamical equations. The most important concept in Dynamical System Analysis is to find out critical points of a set of first-order ordinary differential equations.The application of Dynamical System Analysis to cosmology has been widely discussed in literature [29, 30]. 
The interesting feature of DSA approach in cosmology is the fact that the dynamics of homogeneous and isotropic FRW universes is a special case of generalized Lotka- Volterra system where the competitive species are the barotropic fluids filling the Universe [31, 32]. Without coupling between those fluids, Lotka-Volterra formulation offers a simple way to interpret usual FRW cosmological dynamics. At the same time, one can find the investigations of Lotka- Volterra system in biology in which VIM is actively used [33]-[37]. So, we can apply some of those results to the cosmological FRW model. In this paper, we show the way of doing so.

\section{A brief introduction to VIM}

The VIM has been proposed by J.-H. He [18]-[20], and can be successfully applied to the linear, nonlinear, and boundary value problems. Dealing with this method, one has to construct a correction functional by a general Lagrange multiplier. Then, the Lagrange multiplier can be identified optimally using the variational theory. Being different from the other non-linear analytical methods, such as perturbation methods, this method does not depend on small parameters. It is why VIM can find wide application in non-linear problems without linearization or small perturbations. In order to demonstrate a general idea of VIM, we can consider the following general nonlinear equation:

$$
L[u(x)]+N[u(x)]=g(x),
$$

Where $L$ and $N$ are linear and nonlinear operators respectively, and $g(x)$ is a known function. The correct functional for the equation (1) can be given by

$u_{n+1}(x)=u_{n}(x)+\int_{0}^{x} \lambda(s)\left\{L\left[u_{n}(s)\right]+N\left[\tilde{u}_{n}(s)\right]-g(s)\right\} d s$,

Where

$\Omega_{m(1)}(N)=\Omega_{m 0} e^{-\left(1+3 w_{m}\right) N}+e^{-\left(1+3 w_{m}\right) N} \int_{0}^{N}\left[\left(1+3 w_{m}\right) \Omega_{m 0}^{2} e^{-\left(1+3 w_{m}\right) s}-2 \Omega_{m 0} \Omega_{\Lambda 0} e^{2 s}\right] d s$,
$\Omega_{\Lambda(1)}(N)=\Omega_{\Lambda 0} e^{2 N}-e^{2 N} \int_{0}^{N}\left[2 \Omega_{\Lambda 0}^{2} e^{2 s}-\left(1+3 w_{m}\right) \Omega_{\Lambda 0}(s) \Omega_{m 0} e^{-\left(1+3 w_{m}\right) s}\right] d s$,

Is a Lagrange multiplier, that can be identified optimally using the variational iteration method. At this, $\tilde{u}_{n}$ is considered to be a restricted variation which means that $\delta \tilde{u}_{n}=0$. Making the correct functional (2) stationary, one can obtain

$$
\delta u_{n+1}(x)=\delta u_{n}(x)+\delta \int_{0}^{x} \lambda(s)\left\{L\left[u_{n}(s)\right]+N\left[\tilde{u}_{n}(s)\right]-g(s)\right\} d s=\delta u_{n}(x)+\int_{0}^{x} \delta\left\{\lambda(s) L\left[u_{n}(s)\right]\right\} d s .
$$

The stationary conditions, $\delta u_{n+1}(x)=0$, can be derived using integration by parts in equation (3) and noticing that $\delta u_{n}(0)=0$. The Lagrange multipliers can be easily and precisely obtained for linear problems. However, for nonlinear problems, it is not as trivial. The nonlinear terms are treated as restricted variations such that the Lagrange multiplier can be determined as a simpler form.

The importance and the very utility of method is endowed with the choice of assumption of considering even highly nonlinear and complicated dependent variables as restricted variables thereby synchronizing the error occurring due to process of finding solution to equation (1) to its minimum magnitude. Eventually, after $\lambda$ is determined as desired, a proper selective function, may it be a linear or otherwise with respect to equation (1) is assumed as an initial approximation for finding next successive iterative function by equation (2) recursively.

The successive approximations $u_{n+1}(x)$ of the solution will be readily obtained upon using the obtained Lagrange multiplier and by using any appropriate function for $u_{0}(x)$. The zeroth approximation $u_{0}(x)$ may be selected by any function that just meets, at least, the initial and boundary conditions. Therefore by starting from $u_{0}(x)$, the exact solution may be obtained as

$u(x)=\lim _{n \rightarrow \infty} u_{n}(x)$

Thus, in applications of VIM to differential equations, one should undertake the following three steps: (i) establishing the correction functional; (ii) identifying the Lagrange multipliers; (iii) determining the initial iteration. For the convergence criteria and error estimates of the VIM, one can refer the reader to [21]-[23].

\section{FRW cosmological models}

The Einstein's gravitational equations with a constant cosmological term $\Lambda$ can be written as follows

$a_{\text {exact }}(\tau)=\left[\Omega_{m \Lambda} \cdot \sinh ^{2}\left(\frac{3}{2} \tau\right)\right]^{1 / 3}$.

Where for the sake of simplicity we assume that the gravity coupling constant $8 \pi G=1$ and the speed of light $c=1$. All other symbols have their usual meanings in the Riemannian geometry. Considering all kind of matter in the universe as perfect fluids with the energy densities $\rho_{m}$ and pressure $p_{m}$, one can write down the following energy-momentum (EMT) tensors for all matter components 
$T_{i k}^{(m)}=\left(\rho_{m}+p_{m}\right) u_{i} u_{k}-p_{m} g_{i k}$

Where $u_{i}=(1,0,0,0)$ is 4-velocity of the co-moving observer, satisfying $u_{i} u^{i}=1$. The general assumption of standard cosmology is to use the FRW metric as an isotropic and uniform description of the Universe. The line element of FRW space-time has the following form $d s^{2}=d t^{2}-a^{2}(t)\left[\frac{d r^{2}}{1-k r^{2}}+r^{2}\left(d \theta^{2}+\sin ^{2} \theta d \varphi^{2}\right)\right]$

Where $a(t)$ is a scale factor, and $k=1,0,-1$ for a closed, spatially-flat, open universe respectively. Given this metric and equation (6) for each component of the total EMT $T_{i k}=\sum_{m} T_{i k}^{(m)}$, we can reduce (5) to the following set of equations:

$3 H^{2}+\frac{3 k}{a^{2}}=\sum_{m} \rho_{m}+\Lambda$,

$\dot{H}+3 H^{2}+\frac{k}{a^{2}}=-\sum_{m} p_{m}+\Lambda$

Where $H=\frac{i}{a}$ is the Hubble parameter, and the overdot stands for differentiation with respect to cosmic time $t$.

In the case of non-interacting components of matter, the continuity equation for each of them follows from (7) and (8) as:

$\dot{\rho}_{m}+3 H\left(\rho_{m}+p_{m}\right)=0$

From the assumption of barotropic equation of state (EoS) $\rho_{m}=w_{m} p_{m}$ for each components of the matter, the continuity equations (9) with a constant EoS parameters $w_{m}$ can be integrated explicitly, providing

$\rho_{m}=\rho_{m 0} a^{-3\left(1+w_{m}\right)}$

Where $\rho_{m 0}$ is a constant of integration.

Let us consider now a spatially flat model of the Universe. By putting $k=0$ and substituting (10) into the Friedmann equation (7) along with the Hubble parameter, we obtain the following main equation of the model

$$
\dot{a}^{2}=H_{\Lambda}^{2}\left[\sum_{m} \Omega_{m \Lambda} a^{-\left(1+3 w_{m}\right)}+a^{2}\right]
$$

Where $H_{\Lambda}=\sqrt{\frac{\Lambda}{3}}$, and

$$
\Omega_{m \Lambda}=\frac{\rho_{m 0}}{\rho_{\Lambda}}=\frac{\rho_{m 0}}{3 H_{\Lambda}^{2}}
$$

Is the dimensionless density parameter of the $m$-component of matter, and $\rho_{\Lambda}=\Lambda / 3$ is the vacuum energy density. By introducing the dimensionless cosmic time $\tau=H_{\Lambda} t$, we can rewrite the Friedmann equation (11) as

$a^{\prime 2}=\sum_{m} \Omega_{m \Lambda} a^{-\left(1+3 w_{m}\right)}+a^{2}$

Where the prime stands for the derivative with respect to $\tau$. The Friedmann equation (13) is substantially non-linear, excluding the obvious case of quasi-vacuum EoS, $w_{m}=-1$. So, the method for solving this equation proposed in this paper cannot be directly applied, since it assumes the presence of the linear part in the non-linear equation. Therefore, we can provide a differential consequence of equation (13) which is the following second-order equation

$$
a^{\prime \prime}-a=-\frac{1}{2} \sum_{m}\left(1+3 w_{m}\right) \Omega_{m \Lambda} a^{-\left(2+3 w_{m}\right)},
$$

Provided that $a^{\prime} \neq 0$

\section{Solving the FRW models by VIM}

Comparing equation (1) with the main equation (14), we can write down the correction functional (2) as follows

$$
a_{n+1}(\tau)=a_{n}(\tau)+\int_{0}^{\tau} \lambda_{\tau}(s)\left[\frac{d^{2} a_{n}(s)}{d s^{2}}-a_{n}(s)+\frac{1}{2} \sum_{m}\left(1+3 w_{m}\right) \Omega_{m \Lambda} \tilde{a}_{n}(s)^{-\left(2+3 w_{m}\right)}\right] d s,
$$


Where $\tilde{a}_{n}(s)$ is considered to be a restricted variation. By using the stationary condition for the correction functional (15), we get

$\delta a_{n+1}(\tau)=\delta a_{n}(\tau)+\delta \int_{0}^{\tau} \lambda_{\tau}(s)\left[\frac{d^{2} a_{n}(s)}{d s^{2}}-a_{n}(s)\right] d s$

Using integration by parts in equation (16), and noticing that $\delta a_{n}(0)=\delta a_{n}^{\prime}(0)=0$, we can rewrite this equation as follows

$\delta a_{n+1}(\tau)=\delta a_{n}(\tau)+\lambda_{\tau}(s) \delta a_{n}^{\prime}(s)_{\mid s=\tau}-\lambda_{\tau}^{\prime}(s) \delta a_{n}(s)_{\mid s=\tau}+\int_{0}^{\tau}\left[\frac{d^{2} \lambda_{\tau}(s)}{d s^{2}}-\lambda_{\tau}(s)\right] \delta a_{n}(s) d s$

The stationary conditions $\delta a_{n+1}(\tau)=0$ applied to this equation yield the following equations for the Lagrange multiplier

$$
\left\{\begin{array}{c}
\lambda_{\tau}^{\prime}(s)_{\mid s=\tau}-1=0, \\
\lambda_{\tau}(s)_{\mid s=\tau}=0, \\
\lambda_{\tau}^{\prime \prime}(s)-\lambda_{\tau}(s)=0 .
\end{array}\right.
$$

These equations can be readily solved that yields the following Lagrange multiplier

$\lambda_{\tau}(s)=\sinh (s-\tau)$

Then, according to (15) and (18), the successive approximations $a_{n}(\tau)$ of the solution for the main equation (14) can be readily obtained upon using the obtained Lagrange multiplier (18) and by using any appropriate function for $a_{0}(\tau)$ as

$a_{n+1}(\tau)=a_{n}(\tau)+\int_{0}^{\tau} \sinh (s-\tau)\left[\frac{d^{2} a_{n}(s)}{d s^{2}}-a_{n}(s)+\frac{1}{2} \sum_{m}\left(1+3 w_{m}\right) \Omega_{m \Lambda} a_{n}^{-\left(2+3 w_{m}\right)}(s)\right] d s$,

The zeroth approximation $a_{0}(\tau)$ should satisfy the initial conditions for $a(0)$ and $a^{\prime}(0)$. Thus, by starting from $a_{0}(\tau)$, the exact solution for equation (14) can be obtained as

$a(\tau)=\lim _{n \rightarrow \infty} a_{n}(\tau)$

\subsection{The test example of the universe with dust}

Let us consider the case of FRW cosmology with the only form of matter represented by the pressureless dust. The EoS of such a matter is known as $w_{m}=0$. Therefore, the Friedmann equation (13) becomes as follows

$a^{\prime 2}=\Omega_{m \Lambda} a^{-1}+a^{2}$

Or, according to equation (14),

$a^{\prime \prime}-a=-\frac{1}{2} \Omega_{m \Lambda} a^{-2}$

The exact solution to equations (22) and (23) can be readily found in the following form

$a_{\text {exact }}(\tau)=\left[\Omega_{m \Lambda} \cdot \sinh ^{2}\left(\frac{3}{2} \tau\right)\right]^{1 / 3}$.

In order to determine a possible free parameter in the initial approximation $a_{0}(\tau)$, one can compare our approximate solution of equation

(23) with a series expansion of the exact solution (24) in $\exp (-\tau)$ represented by

$a_{\text {exact }}(\tau)=\sqrt[3]{\frac{\Omega_{m \Lambda}}{4}} e^{\tau}\left[1-\frac{2}{3} e^{-3 \tau}-\frac{1}{9} e^{-6 \tau}-\ldots\right]$.

Now on, we consider the VIM of solving equation (23). In this case, equation (20) becomes as follows

$a_{n+1}(\tau)=a_{n}(\tau)+\int_{0}^{\tau} \sinh (s-\tau)\left[\frac{d^{2} a_{n}(s)}{d s^{2}}-a_{n}(s)+\frac{1}{2} \Omega_{m \Lambda} a_{n}^{-2}(s)\right] d s$.

We can start with initial approximation $a_{0}(\tau)=b_{0} e^{\tau}$ where $b_{0}$ is a constant. Then using the iteration formula (26) with $n=0$ and $n=1$, and Maple software, we can obtain the first approximation as follows 
$a_{1}(\tau)=\left(b_{0}-\frac{\Omega_{m \Lambda}}{12 b_{0}^{2}}\right) e^{\tau}+\frac{\Omega_{m \Lambda}}{4 b_{0}^{2}}\left(e^{-\tau}-\frac{2}{3} e^{-2 \tau}\right)$.

In the same manner, the next approximations can be obtained using the iteration formula (26) and the Maple software. Since parameter $b_{0}$ in this solution remains free, it can be specified with the help of some additional conditions. Here we consider only one simple condition as an example. For this end, we equate the coefficients in the first terms of the equations (25) and (27). As a result, we have the following equation $b_{0}-\frac{\Omega_{m \Lambda}}{12 b_{0}^{2}}=\sqrt[3]{\frac{\Omega_{m \Lambda}}{4}}$.

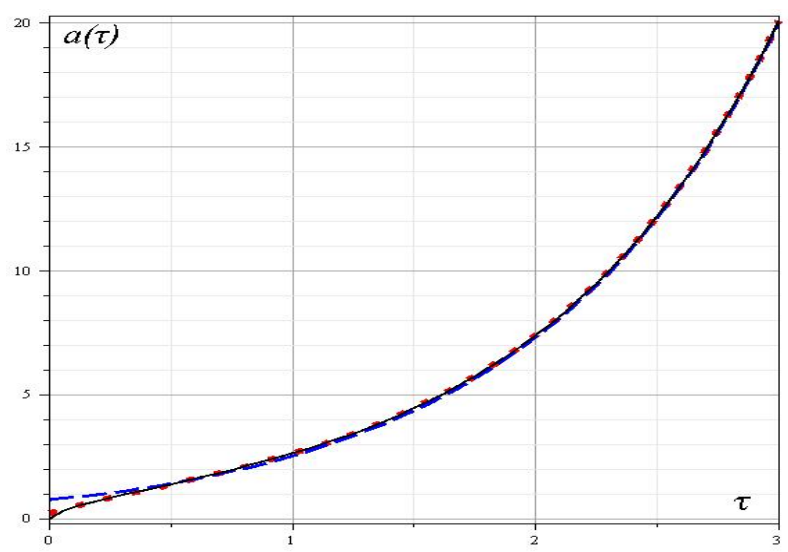

Fig. 1: Shows The Approximate Solutions for the Scale Factor, Given by Equations (25) (Red Line of Points) and (28) (Blue Dashed Line) Compared to the Exact Solution (24) (Black Line), where We Put $\sqrt[3]{\Omega_{m \Lambda} / 4}=1$ for the Sake of Simplicity.

Solving the latter, we have

$b_{0}=\left(\frac{\sqrt[3]{44+12 \sqrt{13}}}{6}+\frac{2}{3 \sqrt[3]{44+12 \sqrt{13}}}+\frac{1}{3}\right) \sqrt[3]{\frac{\Omega}{4}} \approx 1.22285 \sqrt[3]{\frac{\Omega}{4}}$

Substituting this value of $b_{0}$ in equation (27), we get the following approximate solution $a(\tau) \approx a_{1}(\tau)$ :

$a(\tau)=\sqrt[3]{\frac{\Omega_{m \Lambda}}{4}}\left[0.9999 e^{\tau}+0.6687\left(e^{-\tau}-\frac{2}{3} e^{-2 \tau}\right)\right] \approx \sqrt[3]{\frac{\Omega_{m \Lambda}}{4}}\left(e^{\tau}+\frac{2}{3} e^{-\tau}-\frac{4}{9} e^{-2 \tau}\right)$.

The graphical comparison of the approximate solutions (25) and (28) with the exact solution (24) is shown in Fig. 1. Besides, Fig. 2 shows the percentage of relative errors of the approximate solutions compared to the exact one.

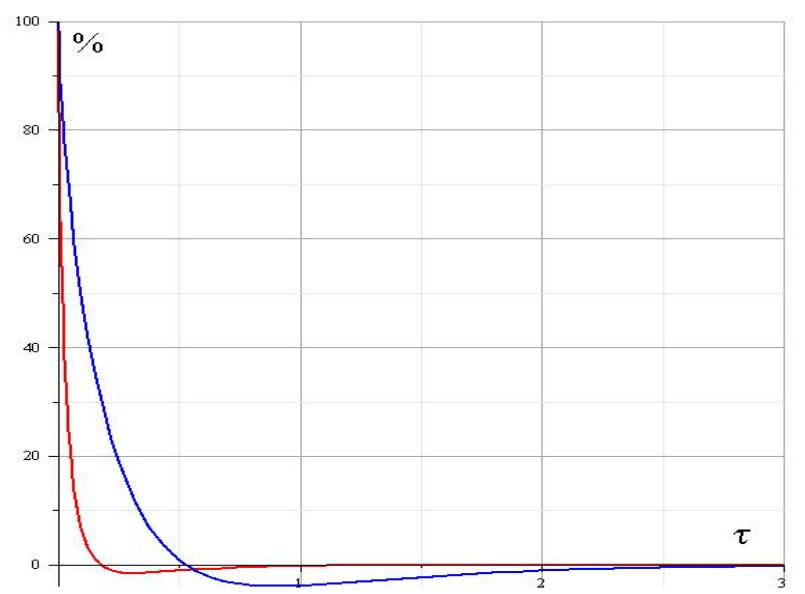

Fig. 2: Shows the Percentage of Relative Errors of the Corresponding Approximations to A (T) Given by Equations (25) (In Red) and (28) (In Blue) Compared to the Exact Solution (24).

\subsection{The model with dust and quintessence}

In this case, we have in equation (14) one more term compared to the previous case. This term is due to a quintessence with equation of state $w_{m}=w_{q}<-1 / 3$. If we consider $w_{q} \geq-1$ in equation (14), then we can obtain the following Friedmann equation instead of (23)

$a^{\prime \prime}-a=-\frac{1}{2} \Omega_{m \Lambda} a^{-2}-\frac{1}{2}\left(1+3 w_{q}\right) \Omega_{q \Lambda} a^{-\left(2+3 w_{q}\right)}$, 
Where $\Omega_{m \wedge}$ is defined by (12), and $\Omega_{q \Lambda}=\frac{\rho_{v 0}}{3 H_{\Lambda}^{2}}$ being the quintessence energy density.

The Friedmann equation (16) can be solved only in quadratures, and does not have an exact analytical solution. So the attempt to solve this equation with the help of VIM, and a comparison of this solution with the corresponding numerical solution are of certain interest.

Therefore, we consider the VIM of solving equation (29). In this case, equation (20) becomes as follows

$a_{n+1}(\tau)=a_{n}(\tau)+\int_{0}^{\tau} \sinh (s-\tau)\left[\frac{d^{2} a_{n}(s)}{d s^{2}}-a_{n}(s)+\frac{1}{2} \Omega_{m \Lambda} a_{n}^{-2}(s)+\frac{1}{2}\left(1+3 w_{q}\right) \Omega_{q \Lambda} a_{n}^{-\left(2+3 w_{q}\right)}(s)\right] d s$.

Once again, we can start with initial approximation $a_{0}(\tau)=b_{0} e^{\tau}$ where $b_{0}$ is a constant. Then using the iteration formula (30) with $n=0$, we can obtain the first approximation $a(\tau) \approx a_{1}(\tau)$ as follows

$a(\tau)=A_{w_{q}} e^{\tau}+B_{w_{q}} e^{-\tau}-C_{w_{q}} e^{-2 \tau}-D_{w_{q}} e^{-\left(2+3 w_{q}\right) \tau}$,

Where

$A_{w_{q}}=b_{0}-\frac{\Omega_{m \Lambda}}{12 b_{0}^{2}}-\frac{\left(1+3 w_{q}\right) \Omega_{q \Lambda}}{12\left(1+w_{q}\right) b_{0}^{2+3 w_{q}}}, B_{w_{q}}=\frac{\Omega_{m \Lambda}}{4 b_{0}^{2}}+\frac{\Omega_{q \Lambda}}{4 b_{0}^{2+3 w_{q}}}, C_{w_{q}}=\frac{\Omega_{m \Lambda}}{6 b_{0}^{2}}, D_{w_{q}}=\frac{\Omega_{q \Lambda}}{6\left(1+w_{q}\right) b_{0}^{2+3 w_{q}}}$.

At this, the condition $\Omega_{m \wedge}+\Omega_{q \wedge}=1$ should be valid.

With the analytical solution for the scale factor (31), we are able to study the model in more detail since almost all kinematics parameters of the model can be expressed by means of the Hubble parameter $H=\frac{d}{a}$. In view of relation $\tau=H_{\Lambda} t$ and equation (31) for the scale factor in our approximation, it is easy to derive that

$H(\tau)=H_{\Lambda} \frac{A_{w_{q}}-B_{w_{q}} e^{-2 \tau}+2 C_{w_{q}} e^{-3 \tau}+\left(2+3 w_{q}\right) D_{w_{q}} e^{-3\left(1+w_{q}\right) \tau}}{A_{w_{q}}+B_{w_{q}} e^{-2 \tau}-C_{w_{q}} e^{-3 \tau}-D_{w_{q}} e^{-3\left(1+w_{q}\right) \tau}}$.

As one can see from (32), the approximate solution (31) is valid for any $w_{q}$, excluding $w_{q}=-1$, and equation (33) shows that $H(\tau \rightarrow \infty)=H_{\wedge}$ regardless of the value of $w_{q} \neq-1$. Moreover, using equations (31)-(33), one can readily verify that $H(\tau=0)=H_{\wedge}$ likewise regardless of the value of $e q \neq-1$.

In the case of $w_{q}=-1 / 3$, the general solution (31) coincides with the scale factor represented by the formula (27). This is a rather obvious result, since the last term in the equation (29), which is proportional to $\Omega_{q \wedge}$, is zero in this case.

For the case of quintessential equation of state $w_{q}=-2 / 3$, we get according to (31)-(33) that

$a(\tau)=\left(b_{0}-\frac{\Omega_{m \Lambda}}{12 b_{0}^{2}}+\frac{\Omega_{q \Lambda}}{4}\right) e^{\tau}-\frac{\Omega_{q \Lambda}}{2}+\left(\frac{\Omega_{m \Lambda}}{4 b_{0}^{2}}+\frac{\Omega_{q \Lambda}}{4}\right) e^{-\tau}-\frac{\Omega_{m \Lambda}}{6 b_{0}^{2}} e^{-2 \tau}$,

And

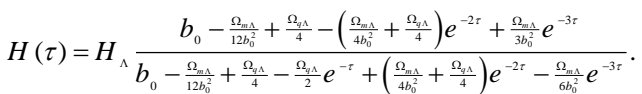

The time behavior of the Hubble parameter in accordance to (33) for some values of $w_{q}$ and $\Omega_{m \Lambda}=0.3, \Omega_{q \Lambda}=0.7$ is shown in Fig. 3 .

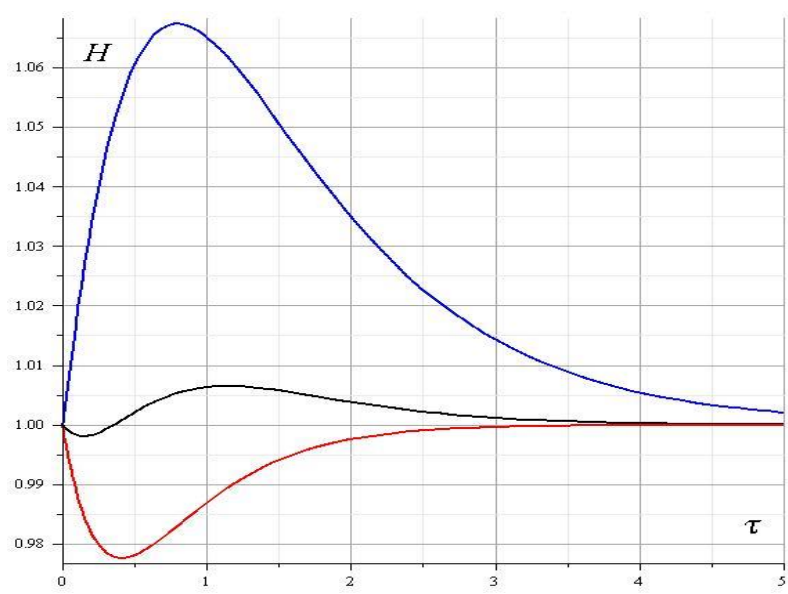

Fig. 3: The Time Behavior of the Hubble Parameter in Accordance to (33) with $H_{\Lambda}=1, b_{0}=1, \Omega_{m \Lambda}=0.3, \Omega_{q \Lambda}=0.7$ and for Different Equation of State: $w_{q}=-1 / 3$ (In Red), $w_{q}=-1.34 / 3$ (In Black) and $w_{q}=-2 / 3$ (In Blue). 
As well known, one of the most important observational parameter in cosmology is the deceleration parameter defined by $q=-\frac{a^{2} a}{a^{2}}=-1-\frac{\dot{H}}{H^{2}}$ where the sign of $q$ indicates whether the model accelerates or not. For a decelerating model we have $q>0$, whereas for an accelerating model of the universe $q<0$. The deceleration parameter $q(\tau)=-1-H_{\Lambda} \frac{H^{\prime}(\tau)}{H^{2}(\tau)}$ can be obtained with the help of equation (33), and it can be expressed by

$$
q=-1+\frac{4 A B e^{-2 \tau}-9 A C e^{-3 \tau}-B C e^{-5 \tau}-9\left(1+w_{q}\right)^{2} A D e^{-3\left(1+w_{q}\right) \tau}+9 w_{q}^{2} C D e^{-3\left(2+w_{q}\right) \tau}-\left(1+3 w_{q}\right)^{2} B D e^{-\left(5+3 w_{q}\right) \tau}}{\left[A-B e^{-2 \tau}+2 C e^{-3 \tau}+\left(2+3 w_{q}\right) D e^{-3\left(1+w_{q}\right) \tau}\right]^{2}},
$$

Where we have simplified all notation for the coefficients (32), omitting the index $w_{q}$. Fig. 4 shows the evolution of $q(\tau)$ as a function of cosmic time $\tau$.

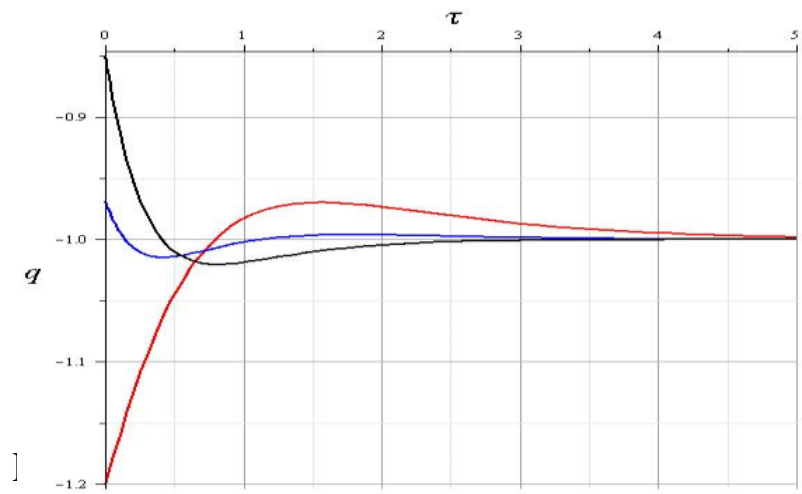

Fig. 4: The Time Behavior of Deceleration Parameter Q of the Model in Accordance to (33) with $H_{\Lambda}=1, b_{0}=1, \Omega_{m \Lambda}=0.3, \Omega_{q \Lambda}=0.7$ and for Different Equation of State: $w_{q}=-1 / 3$ (In Red), $w_{q}=-1.34 / 3$ (In Black) and $w_{q}=-2 / 3$ (In Blue).

\section{Solving FRW model as a jungle universe by VIM}

As known [31, 32t], formally, one can investigate the interesting fact that the dynamics of homogeneous and isotropic FriedmannRobertson-Walker universes is a special case of generalized Lotka- Volterra system where the competitive species are the barotropic fluids filling the Universe. Without coupling between those fluids, Lotka-Volterra formulation offers a simple way to interpret usual FRW cosmological dynamics. A physical coupling between cosmological fluids can be proposed which preserves the structure of the dynamical equations as the standard system of Lotka-Volterra equations. In such a way, we can obtain the system of equations when one of the fluids is coupled to dark energy. This provides a generic behavior for cosmic expansion in presence of coupled species.

In the paper [31], the authors present FRW Universe containing fluids in interaction as a particular case of the well known LotkaVolterra system. This formulation is possible when one considers the system in term of in terms of density parameters for matter

$\Omega_{m}=\frac{8 \pi G}{3 H^{2}} \rho_{m}$,

Evolving through the variable $\ln a$ where $a$ is the scale factor of the Universe. This makes us able to use a lot of standard techniques of dynamical systems analysis in the context of cosmology. Following standard procedure, one can rewrite the set of equations (7)-(9) in terms of density parameters for matter (37), cosmological parameter $\Omega_{\Lambda}$ and curvature $\Omega_{k}$, given by

$\Omega_{\Lambda}=\frac{\Lambda}{3 H^{2}}, \Omega_{k}=-\frac{k}{3 a^{2} H^{2}}$.

Taking into account equations (7)-(9) and (37)-(38), and changing the independent variable to the number of e-folding $N=\ln (a)$, one gets

$\Omega_{m}^{\prime}=\Omega_{m}\left[-\left(1+3 w_{m}\right)+\left(1+3 w_{m}\right) \Omega_{m}-2 \Omega \Lambda\right]$,

$\Omega_{\Lambda}^{\prime}=\Omega_{\Lambda}\left[2-2 \Omega_{\Lambda}+\left(1+3 w_{m}\right) \Omega_{m}\right]$,

Where a prime stands for $N$-derivative, and

$\Omega_{m}+\Omega_{\Lambda}+\Omega_{k}=1$.

The system of equations (39)-(40) formally coincides with the general form of the model of the population dynamics of two species, that is a peculiar Lotka-Volterra system. In the case of the constancy of EoS $w_{m}$, this system coincides up to notations with the system solved by the VIM in [33]. 
The correction functionals of the system (39)-(40) are

$$
\begin{aligned}
& \Omega_{m(n+1)}(N)=\Omega_{m(n)}(N)+\int_{0}^{N} \lambda_{1}(s)\left[\frac{d \Omega_{m(n)}(s)}{d s}+\left(1+3 w_{m}\right) \Omega_{m(n)}(s)\right. \\
& \left.-\left(1+3 w_{m}\right) \tilde{\Omega}_{m(n)}^{2}(s)+2 \tilde{\Omega}_{m(n)}(s) \tilde{\Omega}_{\Lambda(n)}(s)\right] d s, \\
& \Omega_{\Lambda(n+1)}(N)=\Omega_{\Lambda(n)}(N)+\int_{0}^{N} \lambda_{2}(s)\left[\frac{d \Omega_{\Lambda(n)}(s)}{d s}-2 \Omega_{\Lambda(n)}(s)+2 \tilde{\Omega}_{\Lambda(n)}^{2}(s)\right. \\
& \left.-\left(1+3 w_{m}\right) \tilde{\Omega}_{\Lambda(n)}(s) \tilde{\Omega}_{m(n)}(s)\right] d s,
\end{aligned}
$$

Where $\tilde{\Omega}_{m(n)}$ and $\tilde{\Omega}_{\Lambda(n)}$ are considered to be restricted variations with $\delta \tilde{\Omega}_{m(n)}=\delta \tilde{\Omega}_{\Lambda(n)}=0$. From the latter and equations (42)-(43), we obtain

$$
\begin{aligned}
& \delta \Omega_{m(n+1)}(N)=\delta \Omega_{m(n)}(N)+\delta \int_{0}^{N} \lambda_{1}(s)\left[\frac{d \Omega_{m(n)}(s)}{d s}+\left(1+3 w_{m}\right) \Omega_{m(n)}(s)\right] d s, \\
& \delta \Omega_{\Lambda(n+1)}(N)=\delta \Omega_{\Lambda(n)}(N)+\delta \int_{0}^{N} \lambda_{2}(s)\left[\frac{d \Omega_{\Lambda(n)}(s)}{d s}-2 \Omega_{\Lambda(n)}(s)\right] d s .
\end{aligned}
$$

Using integration by parts in equations (44) and (45), we can find out that the Lagrange multipliers $\lambda_{1}(s)$ and $\lambda_{2}(s)$ can be obtained by solving the following system as the stationary conditions:

$$
\begin{aligned}
& \lambda_{1}(s)_{s=N}+1=0,\left.\quad\left[\lambda_{1}^{\prime}(s)-\left(1+3 w_{m}\right) \lambda_{1}(s)\right]\right|_{=N}=0, \\
& \lambda_{2}(s)_{s=N}+1=0,\left.\quad\left[\lambda_{2}^{\prime}(s)+2 \lambda_{2}(s)\right]\right|_{\mid=N}=0 .
\end{aligned}
$$

Therefore, the Lagrange multipliers are

$\lambda_{1}(s)=-\exp \left[\left(1+3 w_{m}\right)(s-N)\right], \quad \lambda_{2}(s)=-\exp [-2(s-N)]$.

Hence, it is follows from equations (42), (43) and (46) that the variational iterations for system (39)-(40) are given by:

$$
\begin{aligned}
& \Omega_{m(n+1)}(N)=\Omega_{m(n)}(N)-\int_{0}^{N} \exp \left[\left(1+3 w_{m}\right)(s-N)\right]\left[\frac{d \Omega_{m(n)}(s)}{d s}+\left(1+3 w_{m}\right) \Omega_{m(n)}(s)\right. \\
& \left.-\left(1+3 w_{m}\right) \Omega_{m(n)}^{2}(s)+2 \Omega_{m(n)}(s) \Omega_{\Lambda(n)}(s)\right] d s, \\
& \Omega_{\Lambda(n+1)}(N)=\Omega_{\Lambda(n)}(N)-\int_{0}^{N} \exp [-2(s-N)]\left[\frac{d \Omega_{\Lambda(n)}(s)}{d s}-2 \Omega_{\Lambda(n)}(s)\right. \\
& \left.+2 \Omega_{\Lambda(n)}^{2}(s)-\left(1+3 w_{m}\right) \Omega_{\Lambda(n)}(s) \Omega_{m(n)}(s)\right] d s,
\end{aligned}
$$

The solution to system (39)-(40) in linearized form (taking $n=0$ ) is as follows:

$\Omega_{m(0)}(N)=\Omega_{m 0} e^{-\left(1+3 w_{m}\right) N}, \quad \Omega_{\Lambda(0)}(N)=\Omega_{\Lambda 0} e^{2 N}$,

Where $\Omega_{m 0}$ and $\Omega_{\Lambda 0}$ are constants of integration. Substituting the initial approximations (49) along with $n=0$ into the system (47) and (48), we have the following equations

$$
\begin{aligned}
& \Omega_{m(1)}(N)=\Omega_{m 0} e^{-\left(1+3 w_{m}\right) N}+e^{-\left(1+3 w_{m}\right) N} \int_{0}^{N}\left[\left(1+3 w_{m}\right) \Omega_{m 0}^{2} e^{-\left(1+3 w_{m}\right) s}-2 \Omega_{m 0} \Omega_{\Lambda 0} e^{2 s}\right] d s, \\
& \Omega_{\Lambda(1)}(N)=\Omega_{\Lambda 0} e^{2 N}-e^{2 N} \int_{0}^{N}\left[2 \Omega_{\Lambda 0}^{2} e^{2 s}-\left(1+3 w_{m}\right) \Omega_{\Lambda 0}(s) \Omega_{m 0} e^{-\left(1+3 w_{m}\right) s}\right] d s,
\end{aligned}
$$

Which after integration yield the first-order approximation:

$$
\begin{aligned}
& \Omega_{m(1)}(N)=\Omega_{m 0} e^{-\left(1+3 w_{m}\right) N}\left(1+\Omega_{m 0}+\Omega_{\Lambda 0}-\Omega_{m 0} e^{-\left(1+3 w_{m}\right) N}-\Omega_{\Lambda 0} e^{2 N}\right), \\
& \Omega_{\Lambda(1)}(N)=\Omega_{\Lambda 0} e^{2 N}\left(1+\Omega_{\Lambda 0}+\Omega_{m 0}-\Omega_{\Lambda 0} e^{2 N}-\Omega_{m 0} e^{-\left(1+3 w_{m}\right) N}\right),
\end{aligned}
$$


The rest of the components of the iteration formulas (47) and (48) can be obtained using the computer algebra package Maple. We demonstrate the time behavior of the energy densities $\Omega_{m(1)}(N)$ and $\Omega_{\Lambda(1)}(N)$ due to equations (50)-(51) for two different equation of state: $w_{q}$ in Fig. 5 .

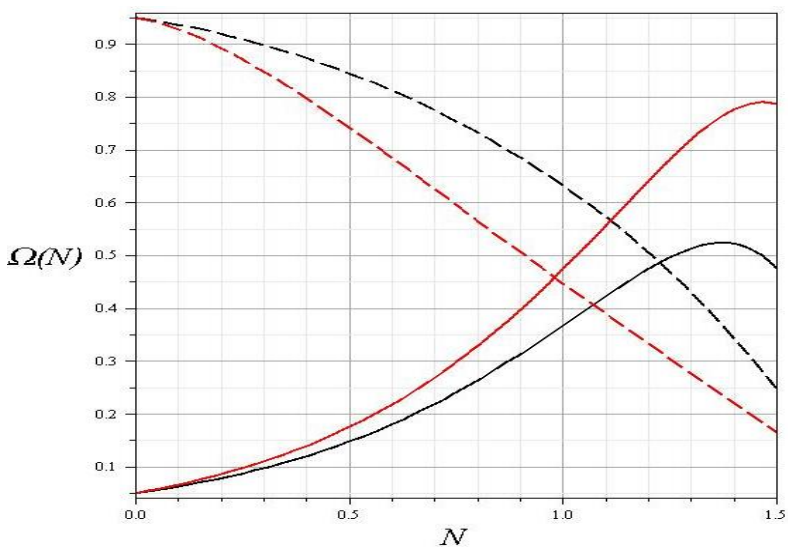

Fig. 5: Shows the Time Behavior of the Energy Densities $\Omega_{m(1)}(N)$ (Dashed Lines) and $\Omega_{\Lambda(1)}(N)$ (Solid Lines) in Accordance to (50)-(51) with $\Omega_{m 0}=0.95$ and $\Omega_{\Lambda 0}=0.05$ for Two Different Equation of State: $w_{q}=0$ (In Red) and $w_{q}=-0.2$ (In Black).

\section{Conclusion}

In this paper, the variational iteration method was employed successfully for solving the Friedmann-Robertson-Walker equation in cosmology. For the illustrative purpose, the exact solution are compared with the approximate solutions in Fig. 1 and Fig. 2 , which show that we can achieve a very good approximation to the exact solution of the Friedmann equation by using only few steps of the iteration formula (20). It is evident that the overall errors can be made smaller by calculating more terms of this sequence.

Further, we have found the approximate solution (31) for the cosmological model of a quintessence field with the equation of state $w_{q}$. Fig. 3 and Fig. 4 show the time behavior of the Hubble parameter $H(\tau)$ and deceleration parameter $q(\tau)$ as the functions of time $\tau$ on the base of the found approximate solution. From these figures and equation (31), one can see the complex behavior of the model, in which the Hubble parameter starts to evolve from the value $H_{\mathrm{A}}$ and asymptotically tends to the same value $H_{\wedge}$ at $\tau \rightarrow \infty$.

Finally, we have explored the DSA approach in cosmology using the fact that the dynamics of the homogeneous and isotropic FRW universes is a special case of generalized Lotka- Volterra system where the competitive species are the barotropic fluids filling the Universe. We have found the iterative equations (47), (48) for the density parameters of the cosmological analog of the generalized Lotka-Volterra set of equations. Fig. 5 illustrates the first-order VIM approximation (50), (51) to the evolution of the matter densities in this model.

The results of this work show that the variational iteration method is a powerful mathematical tool for finding the approximate solutions of the cosmological nonlinear equations.

\section{References}

[1] A. G. Riess, et al., "Observational Evidence from Supernovae for an Accelerating Universe and a Cosmological Constant", Astronomical Journal, Vol. 116 (1998), 1009. https://doi.org/10.1086/300499.

[2] S. Perlmutter, et al., "Measurements of Omega and Lambda from 42 High-Redshift Supernovae", Astrophysical Journal, Vol. 517 (1999), 565. https://doi.org/10.1086/307221.

[3] N. W. Halverson, et al., "Degree Angular Scale Interferometer First Results: A Measurement of the Cosmic Microwave Background Angular Power Spectrum", Astrophys. J., 568 (2002) 38-45. https://doi.org/10.1086/338879.

[4] D. N. Spergel, et al., "First-Year Wilkinson Microwave Anisotropy Probe (WMAP) Observations: Determination of Cosmological Parameters", Astrophys. J. Suppl. Ser., 148 (2003) 175-194. https://doi.org/10.1086/377226.

[5] M. Tegmark, M. A. Strauss, et al., "Cosmological parameters from SDSS and WMAP", Phys. Rev. D, 69 (2004) 103501. https://doi.org/10.1103/PhysRevD.69.103501.

[6] S. W. Allen, R. W. Schmidt, H. Ebeling, et al., "Constraints on dark energy from Chandra observations of the largest relaxed galaxy clusters", Mon. Not. Roy. Astron. Soc., 353 (2004) 457-467. https://doi.org/10.1111/j.1365-2966.2004.08080.x.

[7] L.D. Landau, E.M. Lifshitz. The Classical Theory of Fields, Vol. 2 (3rd ed.), Pergamon Press, 1971.

[8] A. Guth, The Inflationary Universe: A Possible Solution to the Horizon and Flatness Problems, Phys. Rev. D, Vol. $23,347,1981$. https://doi.org/10.1103/PhysRevD.23.347.

[9] A. D. Linde, A New Inflationary Universe Scenario: A Possible Solution of the Horizon, Flatness, Homogeneity, Isotropy and Primordial Monopole Problems, Phys. Lett. B, Vol. 108, 389, 1982. https://doi.org/10.1016/0370-2693(82)91219-9.

[10] E. J. Copeland, A. R. Liddle, D. Wands, "Exponential potentials and cosmological scaling solutions", Physical Review D, 57(8), (1998) 4686-4690. https://doi.org/10.1103/PhysRevD.57.4686.

[11] L. Amendola, "Scaling solutions in general nonminimal coupling theorie", Physical Review D, 60(4) (1999) 043501, https://doi.org/10.1103/PhysRevD.60.043501.

[12] J.-H. He, "Homotopy perturbation technique", Computer Methods in Applied Mechanics and Engineering, 178 (1999), $257-262$. https://doi.org/10.1016/S0045-7825(99)00018-3.

[13] J.-H. He, "A coupling method of homotopy technique and perturbation technique for nonlinear problems", International Journal of Non-Linear Mechanics, 35 (1) (2000), 37-43. https://doi.org/10.1016/S0020-7462(98)00085-7.

[14] V. Shchigolev, "Homotopy Perturbation Method for Solving a Spatially Flat FRW Cosmological Model", Universal Journal of Applied Mathematics, 2(2) (2014), 99-103.

[15] V. Shchigolev, "Analytical Computation of the Perihelion Precession in General Relativity via the Homotopy Perturbation Method", Universal Journal of Computational Mathematics, 3(4) (2015), 45-49.

[16] V. K. Shchigolev, "Calculating Luminosity Distance versus Redshift in FLRW Cosmology via Homotopy Perturbation Method", Gravitation and Cosmology, 23 (2017) 142. https://doi.org/10.1134/S0202289317020098. 
[17] V. K. Shchigolev, D. N. Bezbatko, "Studying gravitational deflection of light by Kiselev black hole via homotopy perturbation method", General Relativity and Gravitation, 51 (2019) 34. https://doi.org/10.1007/s10714-019-2521-6.

[18] J.-H. He, "Variational iteration method - a kind of non-linear analytical technique: some examples", International Journal of Non-Linear Mechanics, 34(4) (1999), 699-708. https://doi.org/10.1016/S0020-7462(98)00048-1.

[19] J.-H. He, "Variational iteration method for autonomous ordinary differential systems", Applied Mathematics and Computation, 114(2-3) (2000), 115-123. https://doi.org/10.1016/S0096-3003(99)00104-6.

[20] J.-H. He, "Variational iteration method-Some recent results and new interpretations", Journal of Computational and Applied Mathematics, 207(1) (2007), 3-17. https://doi.org/10.1016/j.cam.2006.07.009.

[21] M. Tatari and M. Dehghan, "On the Convergence of He's Variational Iteration Method", Journal of Computational and Applied Mathematics, Vol. 207, No. 1, 121-128, 2007. https://doi.org/10.1016/i.cam.2006.07.017.

[22] J. I. Ramos, "On the Variational Iteration Method and Other Iterative Techniques for Nonlinear Differential Equations", Applied Mathematics and Computation, Vol. 199, No. 1, 39-69, 2008. https://doi.org/10.1016/j.amc.2007.09.024.

[23] Ernest Scheiber, "On the Convergence of the Variational Iteration Method", arxiv: 1509.01779.

[24] V. K. Shchigolev, "Variational iteration method for studying perihelion precession and deflection of light in General Relativity", International Journal of Physical Research, 4 (2) (2016) 52-57. https://doi.org/10.14419/ijpr.v4i2.6530.

[25] V. K. Shchigolev, "Analytic Approximation of Luminosity Distance in Cosmology via Variational Iteration Method", Universal Journal of Computational Mathematics, 5(3) (2017) 68-74.

[26] N. Roy, Dynamical Systems Analysis of Various Dark Energy Models. PhD Thesis (2015). arXiv: 1511.07978 [gr-qc].

[27] P. Shah, G.C. Samanta, S. Capozziello, "Qualitative behavior of cosmological models combining various matter fields", Int. J. Mod. Phys. A , 33 (2018) 1850116. https://doi.org/10.1142/S0217751X18501166.

[28] P. Shah, G. C. Samanta, "Stability analysis for cosmological models in $\mathrm{f}(\mathrm{R})$ gravity using dynamical system analysis", The European Physical Journal C, 79(5) (2019), 414. https://doi.org/10.1140/epjc/s10052-019-6934-X.

[29] J. Wainright and G. F. R. Ellis, Dynamical Systems in Cosmology (Cambridge University Press, Cambridge, 1997). https://doi.org/10.1017/CBO9780511524660.

[30] A. A. Coley, Dynamical Systems and Cosmology (Springer, 2003). https://doi.org/10.1007/978-94-017-0327-7.

[31] J. Perez, A. Fuzfa. T. Carletti, L. Melot, L. Guedezounme, "The Jungle Universe: coupled cosmological models in a Lotka-Volterra framework", General Relativity \& Gravitation, 46 (2014) 1753. https://doi.org/10.1007/s10714-014-1753-8.

[32] A. Simon-Petit, H.-H. Yap, J. Perez, "Refinements in the Jungle Universes", http://arxiv.org/abs/1603.02267

[33] B. D. Yuliyanto and S. Mungkasi, "Variational iteration method for solving the population dynamics model of two species", Journal of Physics: Conf. Series, 795 (2017) 012044. https://doi.org/10.1088/1742-6596/795/1/012044.

[34] S. M. Goh, M. S. M. Noorani, I. Hashim, "Introducing variational iteration method to a biochemical reaction model", Nonlinear Analysis: Real World Applications, 11 (2010) 2264-2272. https://doi.org/10.1016/j.nonrwa.2009.06.015.

[35] B. Batiha, M. S. M. Noorani and I. Hashim, "Variational iteration method for solving multispecies Lotka-Volterra equations", Computers and Mathematics with Applications, 54 (2007) 903. https://doi.org/10.1016/j.camwa.2006.12.058.

[36] M. Rafei, H. Daniali and D. D. Ganji, "Variational iteration method for solving the epidemic model and the prey and predator problem", Applied Mathematics and Computation, 186 (2007) 1701. https://doi.org/10.1016/j.amc.2006.08.077.

[37] F. Shakeri and M. Dehghan, "Numerical solution of a biological population model using He's variational iteration method", Computers and Mathematics with Applications, 54 (2007) 1197. https://doi.org/10.1016/j.camwa.2006.12.076. 